\title{
Laser-induced Black-body Heating (LIBBH) as a Method for Glass Surface Modification
}

\author{
Roman Zakoldaev, Maksim Sergeev, Galina Kostyuk and Vadim Veiko \\ ITMO University, 197101, 49 Kronverkskiy av., St. Petersburg, Russia, e-mail: zakoldaev@gmail.com
}

\begin{abstract}
The technique for laser-assisted modification of glass surface is suggested. The method is based on the heating of glass by graphite plate irradiated by laser being in direct contact with the glass surface from the backside. Transparency of glass and strong absorption of graphite for an incident laser radiation provide the diversity of applicable lasers wavelength and pulse duration. In our case, the pulsed fiber NIR laser was used. The surface densification of porous glass as well as creation of various micro-optical elements on the quartz glass surface are reported.
\end{abstract}

DOI: $10.2961 /$ jlmn.2015.01.0003

Keywords: Laser micromachining, porous glass, densification, nanocomposite, MOE

\section{Introduction}

Glass microstructuring is one of the trends in the development of modern laser technology. Micro-optical elements (MOE) are used in optical communication systems [1], high-quality imaging systems [2] or in adaptive optics [3], its creation is based on these technologies. In laser systems, such MOE are usually used for homogenizing of laser beams $[4,5]$ and for laser beam splitting $[6,7]$.

In most cases, MOE are created on the surface of different materials for technology and applications, which is dialed with visible and near IR spectral range. As a result, developers' preference is given to silicate glass. It implies the usage as a quartz glass, porous glass (PG) or quartzoid glass. In previous years, we used $\mathrm{PG}$ as a glass material to create MOE on the surface [8] and in the bulk of material [9].

Unfortunately, the "ageing" process is quite typical for PG [10]. Because of that fact, the optical and physicchemical properties can change due to the absorption of water, carbon dioxide and organic compounds from the air. This process is shown in a reduction of samples transmission, in a shift of optical spectra, etc. Besides, stabilization of the optical characteristics of PG is important for creation nanocomposites matrices produced by impregnation of $P G$ with different impurities for different photonics purposes $[11,12]$.

The main point, that all development technologies have many restrictions. In such manner, forming of MOE on the glass surface were implemented under the action of $\mathrm{CO}_{2}$ laser radiation $[13,14]$ and $\mathrm{UV}$ radiation. These wavelengths corresponds to the fundamental absorption on the $\mathrm{Si}-\mathrm{O}$ bonds for silicate glasses. Lasers with femtosecond pulse duration are integral part in the technology of glass micromachining [15]. These technologies are based on multiphoton absorption and following transformation processes.

There are some other technologies, which mostly free of the restrictions on the applied types of lasers. The closest analogs are a back laser-induced film transfer (back LIFT) $[16,17]$, laser-induced backside dry etching (LIBDE) $[18,19]$ and laser induced plasma assisted ablation (LIPPA)
[20,21]. All of these approaches are based on the strong absorption of laser radiation by a solid media placed under the transparent material.

In this article, a laser technology for surface glass modification - lased-induced black-body heating (LIBBH) is reported. It allows using any required laser with wavelength, which is transparent for the glass. The method is based on the heating of glass by expended graphite (Gr) plate being in direct contact with the backside of the glass surface and irradiated by laser beam. The difference of this method with LIBDE is the choice of another, much more efficient heater, because graphite has a higher absorbance, which does not depend on the wavelength of an incident radiation. As for LIPAA the configuration of the LIBBH is much more optically simple and more suitable for the computer control of the pictures topology.

There are two important applications: first one is prevention of PG "ageing" process by densification of the surface layer of PG and second is MOE creation on a quartz and other glasses surface.

\section{Experimental details}

The main objects of the investigation were planeparallel plates of $\mathrm{PG}$ with the thickness of $1.5 \mathrm{~mm}$. Formation of MOE was carried out on a surface of planeparallel plates of polished quartz glass with the thickness of $1.5 \mathrm{~mm}$.

Figure 1 shows schematically the experimental setup. We used ytterbium fiber laser with wavelength of $1.07 \mu \mathrm{m}$, pulse duration of 100-200 ns, pulse repetition rate of 20 $100 \mathrm{kHz}$ as a radiation source. Laser beam was scanned with a two-coordinate galvanometer scanner based on drivers G325DT «GSI Lumonics». Laser radiation (1) goes through optical fiber (2) and leads into the collimator (3) expanding the laser beam from $50 \mu \mathrm{m}$ to $8 \mathrm{~mm}$. Then laser beam moves along axes $\mathrm{X}$ and $\mathrm{Y}$ by mirror of scanning system (4) and (5), which are rotated with stepper motors (6) and (7). After a scanning system laser beam is focused by the objective lens (the focal length is $210 \mathrm{~mm}$ and the size of a focal spot is $55 \mu \mathrm{m})(8)$ on the boundary between glass (9) and Gr plate (10) which were fixed on the table (11). The galvanometer system (4-7) and parameters of 
laser radiation were controlled by a personal computer (12). It is important to note, that all types of used glass plates are completely transparent for the incident radiation and the absorptivity of the expended graphite in a wide range of wavelengths is close to 1 [22].

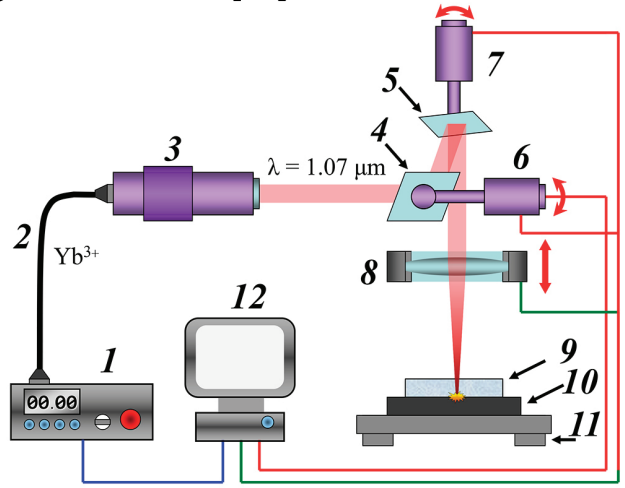

Fig. 1 Experimental setup for glass surfaces microstructuring.

PG densification was carried out through step by step scanning of a laser beam at a speed $V=300 \mathrm{~mm} / \mathrm{s}$, pulse repetition frequency $v=20 \mathrm{kHz}$, average power $P=10 \mathrm{~W}$ and pulse duration $\tau=100 \mathrm{~ns}$. The overlap factor was $98 \%$. Optimum characteristics of PG densification and MOE formation on the quartz glass surface are shown at the Table 1.

Table 1 Optimum parameters of different LIBBH modes

\begin{tabular}{c|c|c|c|c}
\hline & $\mathrm{P}, \mathrm{W}$ & $\mathrm{f}, \mathrm{kHz}$ & $\tau, \mathrm{ns}$ & $\mathrm{V}, \mathrm{mm} \cdot \mathrm{s}^{-1}$ \\
\hline PG densification & $10-11$ & $20-30$ & 200 & 500 \\
MOE formation & $13-15$ & $20-50$ & 100 & 300 \\
Cleaning & 8 & 20 & 200 & 3500 \\
\hline
\end{tabular}

After laser machining, glass surface were always coated by graphite particles. The cleaning of a glass plate was made on the same experimental setup, but with other regime of laser machining. Laser radiation was focused on contaminated structured surface of a glass, coated with thin water layer. The optimum characteristics of the laser irradiation at a cleaning were determined experimentally (Table 1).

PG with densification layers and MOE on the quartz glasses surface were studied using an optical microscope (Carl Zeiss Axio). The morphology of the modified glass was investigated using an atomic-force microscope (Solver PRO-M) and profilometer (Hommel Tester T8000).

\section{Results and discussion}

\subsection{Porous glass surface densification}

As a result of the experiments, thin densification layers were formed consecutively on the both sides of the PG. With optimum characteristics of laser irradiation, we managed to form densificated layers without cracks. In Figure 2 face view pictures of PG surface with a densificated layer are shown. The measured thickness of the densificated layer $h_{d}$ was approximately 5-10 $\mu \mathrm{m}$. Actually, a shallow thick- ness was realized by short pulse duration and by main factor of this technology, connected with the heat transfer from a graphite plate to PG surface. The thickness of a heated layer can be estimated as $h_{d}=(a \tau)^{1 / 2}$, where $a-\mathrm{PG}$ thermal conductivity $\left(a \sim 1.24 \cdot 10^{-6} \mathrm{~m}^{2} \cdot \mathrm{s}^{-1}\right), \tau$ - pulse duration time. At $\tau=100$ ns the thickness of a heated layer could reach $h_{T}=0.35 \mu \mathrm{m}$. Probably, the difference between experimental and calculated results is due to multi-pulse laser processing. Besides, it may be connected with a mechanical action of the graphite particles, which are due to an effervescence and possibly burning of graphite.

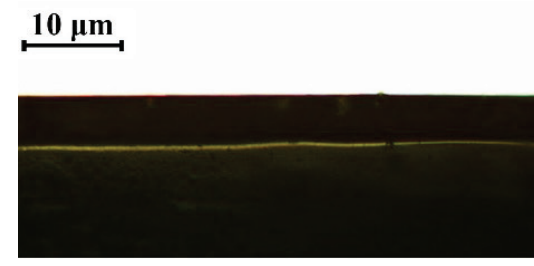

Fig. 2 Face view of PG surface after LIBBH.

Surface topography of a PG plate before and after LIBBH (including laser cleaning) are shown in Figure 3(a) and 3(b) correspondingly. This figure shows that the pores were totally closed after LIBBH.

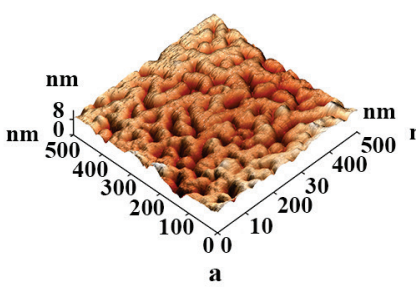

Fig. 3 Atomic force microscope topographical scan of PG surface before (a) and after LIBBH (b).
To control a PG surface densification quality impregnation of PG with glycerol was used. PG samples were being impregnated during 72 hours. One of them was the subject to LIBBH. It is known, that transmission is highly affected during the 'ageing' process. That's why, we carried out micro spectrophotometric research of a PG transparency before and after LIBBH. Transmission spectrum is shown in Figure 4 and it can be clearly seen that densified layers keep its transmission with 1-5\% fluctuations during 10 months.

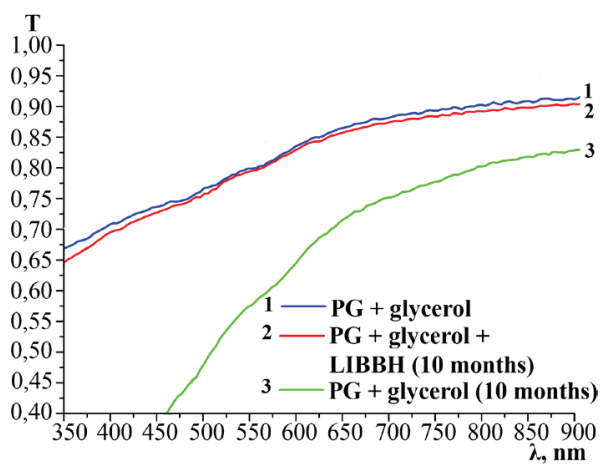

Fig. 4 Transmission spectrum of a PG plate. 
The investigation of PG with densified layers shows the fact of long-term stabilization of PG optical properties. Besides, this technology allows preserving a pore structure in between densified layers.

\subsection{LIBBH for formation of microlenses array}

The other possible application of LIBBH method is formation of microlenses arrays (MLA) on quartz glass surface. The control of microlense location and size was made in accordance with a template previously developed on the personal computer. The template was designed in such a way that the formation of microlenses occurred at those segments of a glass surface, where laser radiation was blocked.

Table 2 Geometrics and optical characteristics of microlenses

\begin{tabular}{c|c|c|c|c}
\hline$d_{M}, \mu \mathrm{m}$ & $h_{M}, \mu \mathrm{m}$ & $R_{c}, \mu \mathrm{m}$ & $f_{e}, \mu \mathrm{m}$ & $d_{\min }, \mu \mathrm{m}$ \\
\hline 70 & $5.1 \pm 0.1$ & 122.65 & $260 \pm 10$ & $6.0 \pm 0.1$ \\
150 & & 908.81 & $1970 \pm 10$ & $20.2 \pm 0.1$ \\
250 & $3.1 \pm 0.1$ & 2521.71 & $5480 \pm 10$ & $31.5 \pm 0.1$ \\
450 & & 8166.87 & $17750 \pm 10$ & $62.3 \pm 0.1$
\end{tabular}

In this experiment MLA with a size of 10x10 mm and with various dimensions and period of microlense were formed. In particular, when diameter of microlense $d_{M}=70$ $\mu \mathrm{m}$ and period of placing $t=140 \mu \mathrm{m}$, number of MOE was $N=2116$. The measurements of microlense focal lengths $\left(f_{\boldsymbol{e}}\right)$ and focal spot diameters $\left(d_{\min }\right)$ were made according with a technique as in the work [9]. The microlense focal spot diameter was measured on $1 / \mathrm{e}^{2}$ level. All geometric and optical characteristics of microlenses are listed in the Table 2. Moreover, calculated results of focal lengths $\left(f_{c}\right)$ based on knowledge of microlenses geometric characteristics and refractive index $(n=1.46$, for $\lambda=0.635 \mu \mathrm{m}$ was used in experiment) is shown in the table. The diameter of focal spot size $\left(d_{\text {minc }}\right)$, calculated according to the Rayleigh criterion is also shown. Agreement of measured and calculated are in good correspondence.

Figure 5 shows the topography of single microlense $\left(d_{M}=70 \mu \mathrm{m}\right)$ on a quartz glass plate. Follow to given data the sag of MOE $h_{M}$ is $5.1 \pm 0.1 \mu \mathrm{m}$ as a result the calculated radius of microlense $R_{c}$ is $122.65 \mu \mathrm{m}$. From this figure it can be found that the roughness $R_{a}$ of microlense top corresponds to a roughness of polished quartz glass $\left(R_{a}=0.01\right.$ $\mu \mathrm{m})$. At the same time, the roughness of MOE surrounding area has a value of approximately $1 \mu \mathrm{m}$. Probably it happens due to an influence of graphite particles with high temperature and speed.

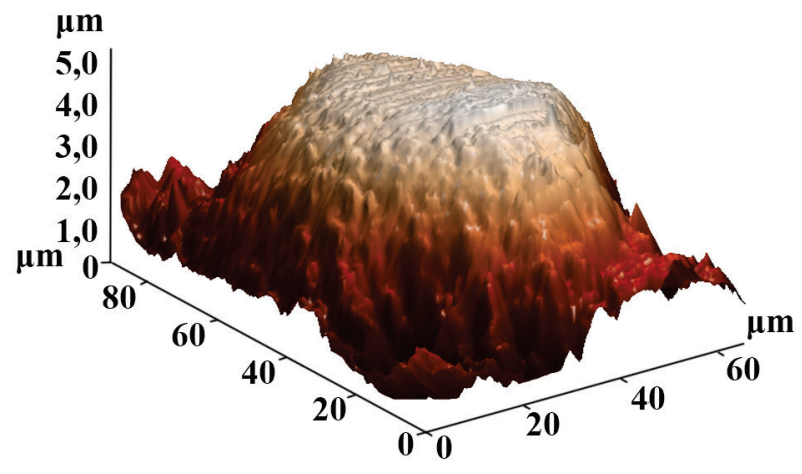

Fig. 5 Atomic force microscope topographical scan of microlense,

$$
d_{M}=70 \mu \mathrm{m} \text {. }
$$

\subsection{LIBBH for microstructuring}

It is known, that MOE structures are divided into two types: periodical MOE worked on the Fraunhofer diffraction principle, and typical MOE based on the laws of refraction and reflection of light. Periodical MOE are used as phase diffraction gratings (PDG), which are able to create a high and equal intensity of the first diffraction orders. It is necessary for a simultaneous laser processing of a surface [23]. Besides, they are used as random phase plates (RPP), which are already proven as homogenizers for multimode laser radiation with an inhomogeneous structure of the beam [24].

The PDG with a period $p=30-100 \mu \mathrm{m}$, ratio $S / p=0.30$ 0.55 (where $S$ - the size of the modified lines) were formed on the surface of quartz glass. The RPP with elements of square shape with a size $d_{R P P}=200-700 \mu \mathrm{m}$ and situated in random manner were formed either. The depth of all elements were in the range of $h=400-1200 \mathrm{~nm}$.

Figure 6 shows photographs of the fragments made by optical microscope: (a) - the PDG ( $p=100 \mu \mathrm{m}$, ratio $S / p=$ $0.5),(\mathrm{b})-$ the RPP $\left(d_{R P P}=250 \mu \mathrm{m}\right)$. Figure $6(\mathrm{c})$ and $(\mathrm{d})$ are photographs of the same fragments made in a linearly polarized light with crossed polarizer and analyzer. Dark background of both images indicates the absence of thermomechanical stresses around both microstructures.

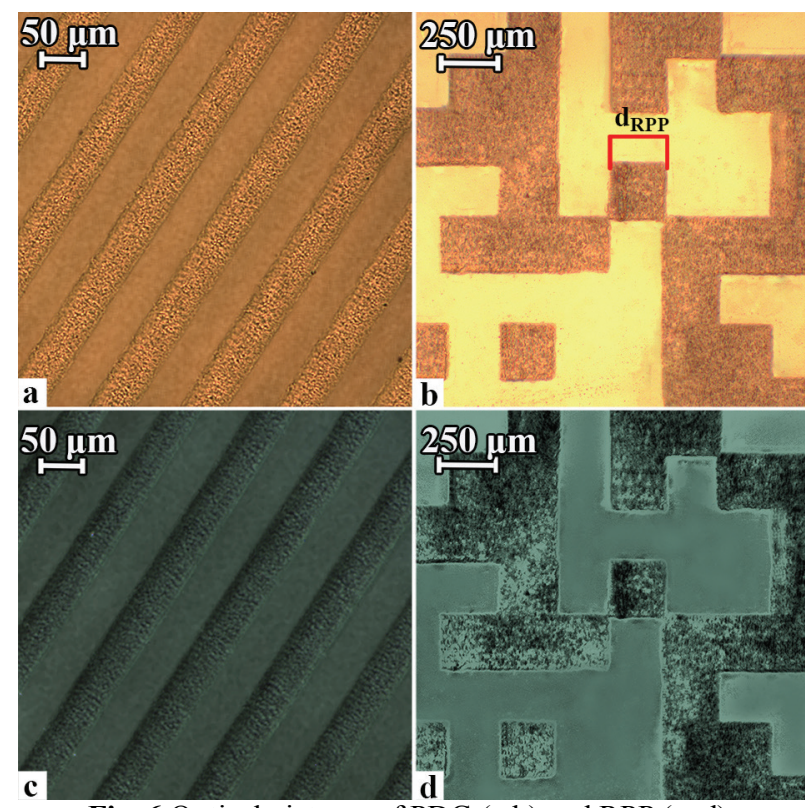

Fig. 6 Optical pictures of PDG (a,b) and RPP (c, d). 


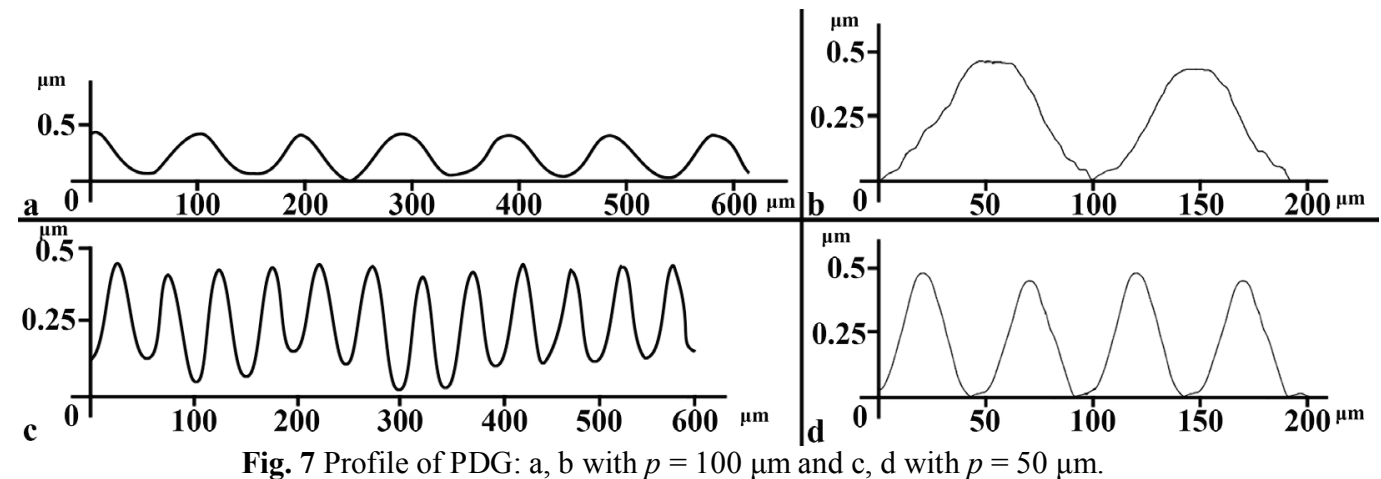

Profile of PDG is shown in Figure 7. Roughness $R_{a}$ of PDG surface is several times higher in comparison with the basic material. The roughness of microstructures, which were created by our technology are slightly higher than the value of $R_{a}$, which were reported for LIBDE technology.

Fabricated PDG were tested for simultaneous laser processing of thin titanium films on a substrate of glass BK-7. Experimental setup is shown in Figure 8(a). We used fiber laser radiation with $\lambda=1.07 \mu \mathrm{m}$ (1), the radiation beam is expanded by a telescopic system (2) with $4^{\mathrm{x}}$ increasing. PDG (3) $(p=100 \mu \mathrm{m}$, ratio $S / p=0.5$ and depth $h=450$ $\mathrm{nm}$ ) was placed behind the telescopic system (2). Aperture (4) with size $\left(d_{\text {apert }}=2 \mathrm{~mm}\right)$ was used to remove a high diffraction orders with a low intensity. Convex lens (5) $\left(d_{1}\right.$ $=60 \mathrm{~mm}, f=300 \mathrm{~mm}$ ) was placed behind the diaphragm (4). The titanium film on the glass substrate (6) with film thickness $h_{T i}=60 \mathrm{~nm}$ was used as a target for laser micromachining.

Figure 8(b) shows three holes in a titanium film made by PDG laser simultaneous processing in the focal plane of the convex lens. Laser processing parameters were as follows: laser power $P_{y t}$ was $1 \mathrm{~W}$ and exposure time $\tau=1 \mathrm{~s}$.

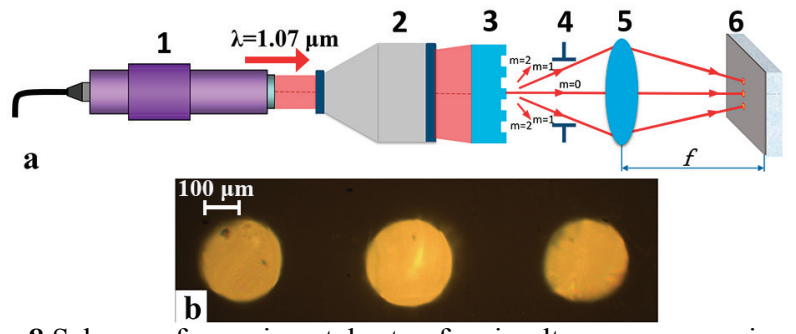

Fig. 8 Scheme of experimental setup for simultaneous processing of a titanium film (a); picture of three holes made on the Ti film by PDG laser simultaneous processing (b).

Three holes were made during a single exposure (Fig. 8(b)). A smooth outline border of holes shows the success of the experiment on simultaneous processing of the Ti film. PDG usage was reported in [14], where some advantages were marked in comparison with MLA. First, MLA have spaced regions between microlenses, which create an additional flash exposure of a machining plane. Besides, in most cases laser beam has a Gaussian distribution of intensity profile, as a result incident radiation irregularly illuminates MLA. In such a manner, design and production of PDG, which can create equal and small spots, i.e. suitable for simultaneous processing of materials.

\subsection{Physical sense of LIBBH discussion}

To explain above results for PG and quartz glass laser processing it is necessary to take into account not only conductive heating of glass due to a heat contact with graphite plate. The maximum temperature at the surface of graphite in the center of irradiation zone can be estimated as [25]:

$$
T_{c}=\frac{2 A_{c} q_{0} \sqrt{a_{c} \tau}}{k_{c} \sqrt{\pi}}+T_{i},
$$

where $A_{c}$ - absorptance of expended graphite $\left(A_{c} \sim 1.0\right), a_{c}$ thermal conductivity of expended graphite $\left(a_{c}=1.24 \cdot 10^{-4}\right.$ $\left.\mathrm{m}^{2} / \mathrm{s}\right), k_{c}$ - heat diffusion of the graphite $\left(k_{c}=2000\right.$ $\mathrm{W} /(\mathrm{m} \cdot \mathrm{K})), \tau$ - pulse duration (100 ns), $T_{i}$ - initial temperature $\left(T_{i}=298 \mathrm{~K}\right)$ and $q_{0}$ - laser power density at the focal spot $\left(q_{0} \sim 7,7 \cdot 10^{7} \mathrm{~W} / \mathrm{cm}^{2}\right.$ with average laser power $8 \mathrm{~W}$ and pulse repetition rate $20 \mathrm{kHz}$ ).

At pointed data $T_{c}$ can reach up to $\sim 4.8 \cdot 10^{3} \mathrm{~K}$ and even more. Optical observations and spectral measurements of an erosion plasma partially confirm this estimation. At such high temperatures, a number of different transformations take place with an expended graphite and two of them are the most important: 1) cellular graphite appearance during laser micromachining process and 2) fast burning of carbon in the air at its heating. Both processes noticeably activate at $T$ more than $1000 \mathrm{~K}$ [22]. In any case, it leads to a shock mechanical action on a softened surface of glass. The confirmation of an important role of the shock mechanical action at this process is the fact that no any structure modification happens in the absence of the strong graphite vapors jet (densification regime - Fig.9) or plasma plume (modification regime).

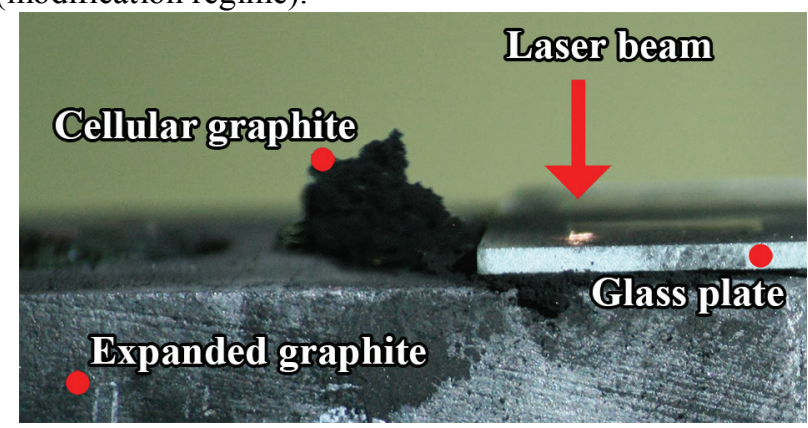

Fig. 9 Photo of cellular graphite effervescence during a laser micromachining.

\section{Conclusion}

LIBBH method for glass processing allows realizing different modes of laser treatment: for local densification to surface microstructuring and microoptical components 
fabrication. The possibility of formation of thin densified layer on a PG surface is experimentally demonstrated. It stabilizes optical characteristics of PG, i.e. prevents "ageing" process. Besides, porous structure in between densified layers was saved.

MLA with different fill factors and dimensions were formed on a quartz glass surface by LIBBH. The MLA time of formation depends on an array size and usually takes 515 min. Determining of MOE focal length was performed. Numerical aperture of microlenses $N A$ depends on the element size, and is equal to $0.02-0.13$.

Finally, microstructuring of glass surfaces as well as PDG and RPP structures formation was experimentally demonstrated. The depth of such structures were in the range $h=400-1200 \mathrm{~nm}$, depending on the laser beam parameters. The PDG was chosen for laser simultaneous processing of a titanium film.

We believe that deeper LIBBH investigation skills and using of different lasers can open new opportunities for its applications.

\section{Acknowledgments} 00351.

The study was supported by RSF agreement № 14-12-

\section{References}

[1] D.A.B. Miller: Proc. of the IEEE, (2009), 97(7), p. 1166

[2] Hsin-Ta Hsieh, Vinna Lin, Jo-Lan Hsieh, Guo-Dung John Su: Optics Communications, 284, (2011) 5225.

[3] M.M. Vekshin, A.S. Levchenko, A.V. Nikitin, V.A. Nikitin, N.A. Yacovenko: Meas. Sci. Technol., 21, (2010) 1.

[4] C. Kopp, L. Ravel, P. Meyrueis: J. of Optics A: Pure and Applied Optics, 1, (1999) 398.

[5] R. Gediminas, S. Evaldas, G. Paulius, G. Mindaugas, B. Christian, J. Erwin, U. Udo and V. Friedemann: J. of Laser Micro/Nanoengineering, 61, (2011) 1.

[6] D. Nieto, G. Vara, J. Diez, G. O’Connor, J. Arines, C. Gómez-Reino and M. Flores-Arias: J. of Micro/Nanolithography, MEMS, and MOEMS, 11, (2012) 023014-1.

[7] R. J. Winfield, B. Bhuian, S. O'Brien, and G.M. Crean: Appl. Surf. Sci., 253, (2007) 8086.

[8] T.V. Antropova, V.P. Veiko, G.K. Kostyuk, M.A. Girsova, I.N. Anfimova, V.A. Chuiko, E.B. Yakovlev: J. Glass Physics and Chemistry, 38(6), (2012) 478.

[9] R.A. Zakoldaev, M.M. Sergeev, G.K. Kostyuk, E. B. Yakovlev: J. Glass and Ceramics, 70(11-12), (2014) 395.

[10]T.V. Antropova, I.A. Drozdova: J. Optica Applicata, 33(1), (2003) 13.

[11] L. Yang, M. Yamashita, T. Akai: Optics Express, 17, (2009) 6688.

[12] A. Burchianti, A. Bogi, C. Marinelli, E. Mariotti and L. Moi: Optics Express, 16, (2008) 1377.

[13] V.P. Veiko, E.B. Yakovlev, A.J. Nikiphorov, V.A. Chuiko: "Laser methods of controlling porous silica glass structure" ed. By L.L. Hench and J.K. West, (Publisher, Wiley, New York, 1992) p.919.
[14] M. Wakaki, Y. Komachi, G. Kanai: Appl. Opt., 37, (1998) 627.

[15] J. Ihlemann, B. Wolff, P. Simon: Appl. Phys. A, 54, (1992) 363

[16] V. P. Veiko, E.A. Shakhno, B.M. Yurkevich, V.A. Chujko, B.G. Podlaskin and N.A. Tokranova: Photonics West'96. - SPIE, (1996) 541.

[17] V. P. Veiko, E.A. Shakhno, V.N. Smirnov, A.M. Miaskovski and G.D. Nikishin: Laser and Particle Beams , 24(02), (2006) 203.

[18] B. Hopp, C. Vass, T. Smausz, and Z. Bor: J. of Physics D: Applied Physics, 39 (22), (2006) 4843.

[19] B. Hopp, C. Vass, and T. Smausz: Appl. Surf. Sci., 253(19), (2007) 7922.

[20] J. Zhang, K. Sugioka and K. Midorikawa: Appl. Phys. A, 67, (1998) 499.

[21] K. Sugioka, K. Obata, M.H. Hong, D.J. Wu, L.L. Wong, Y.F. Lu, T.C. Chong and K. Midorikawa: Appl. Phys. A 77, (2003) 251.

[22] A.S. Fialkov: Carbon. Interlayer compounds and compositions thereof. (Publisher, Moscow, 1997), p. 345 (in Russian).

[23] R.J. Winfield, B. Bhuian, S. O'Brien and G.M. Crean: Appl. Surf. Sci., 253, (2007) 8086.

[24] Y. Kato, K. Mima, N. Miyanaga, S. Arinaga, Y. Kitagawa, M. Nakatsuka and C. Yamanaka: Physcal Review Letters, 53, (1984) 1057.

[25] S.M. Metev, V.P.Veiko: "Laser-assisted microtechnology" (Publisher, Springer Verlag, 1994).

(Received:June 19, 2014, Accepted: November 28, 2014) 